\title{
Genetic basis of incidence and period length of circadian rhythm for locomotor activity in populations of a seed beetle
}

\author{
T Harano and T Miyatake
Laboratory of Evolutionary Ecology \\ Laboratory of Evolutionary Ecology, Graduate School of Environmental Science, Okayama University, Okayama, Japan
}

\begin{abstract}
Circadian rhythms are ubiquitous in a wide variety of organisms, although their genetic variation has been analyzed in only a few species. We found genetic differences in the circadian rhythm of adult locomotor activity among strains of the adzuki bean beetle, Callosobruchus chinensis, which differed in origin and have been maintained in isolation. All beetles in some strains clearly had free-running rhythms in constant darkness whereas most beetles in other strains were arrhythmic. The period of freerunning rhythm varied from approximately 19 to $23 \mathrm{~h}$ between the strains. $F_{1}$ males from reciprocal crosses among strains with different periods of circadian rhythms had circadian periods that
\end{abstract}

Keywords: bean beetle; Callosobruchus; circadian clock; free-running period; genetic variation; locomotor activity

were intermediate between their parental strains. Segregation of the circadian rhythm appeared in the $F_{2}$ generation. These findings are consistent with the hypothesis that variation in the period length of circadian rhythm is explained by a major autosomal gene with additive effects and no dominance. This hypothesis was supported by the joint scaling test for the free-running period in the $F_{1}$ and $F_{2}$ generations. We discuss possible causes for genetic variation in circadian rhythm in the C. chinensis strains in terms of random factors and selection. Heredity (2010) 105, 268-273; doi:10.1038/hdy.2010.4; published online 10 February 2010

\section{Introduction}

Endogenous circadian clocks, which produce rhythms in biological processes with a period close to a day, are ubiquitous in a wide variety of organisms, from bacteria to humans (for example, Edmunds, 1988; Panda et al., 2002). Circadian rhythms relating to physiological and behavioral activities have been measured in many insects. Many such reports have included speciesspecific periods of circadian rhythm (Saunders, 2002). In contrast, studies of intra-specific variation in circadian rhythms, including genetic variation, have majored on model organisms for chronobiology. Among insects, Diptera, especially Drosophila melanogaster, predominate in studies of the genetics of circadian rhythm (Hall, 2003).

In a landmark study of circadian rhythm, period (per) mutants were isolated from $D$. melanogaster flies exposed to a chemical mutagen (Konopka and Benzer, 1971). It was found that the per mutation, which is located at one locus on the $X$ chromosome, altered the normal 24-h period of circadian rhythms of eclosion and adult locomotor activity. In one mutant $\left(p^{s} r^{s}\right)$, this period shifted to $19 \mathrm{~h}$, where in another $\left(p e r^{\mathrm{L}}\right)$, it had shifted to $29 \mathrm{~h}$ and in the third $\left(p^{0} r^{\circ}\right)$, it became arrhythmic (Konopka and Benzer, 1971). As a result of this study,

Correspondence: Current address: Dr T Harano, Laboratory of Ecological Science, Department of Biology, Faculty of Sciences, Kyushu University, 6-10-1 Hakozaki, Higashi-ku, Fukuoka 812-8581, Japan.

E-mail: tharano518@yahoo.co.jp

Received 11 June 2009; revised 12 December 2009; accepted 23

December 2009; published online 10 February 2010 mutants altering circadian rhythms have now been found in various other model organisms such as Chlamydomonas (Bruce, 1972), Neurospora (Feldman and Hoyle, 1973), hamsters (Ralph and Menaker, 1988), mice (Vitaterna et al., 1994), cyanobacteria (Kondo et al., 1994) and Arabidopsis (Millar et al., 1995). Moreover, per homologs have been identified in a variety of animals (for example, Reppert et al., 1994; Tei et al., 1997; Hall, 2003; Matsumoto et al., 2008). Thus, genetic variation in circadian rhythm may be common in animals, although, thus far, it has only been detected in a limited number of species.

Another approach to the search for genetic variation in circadian rhythm is the screening of spontaneous variation between different populations. Geographical variation in the period length of circadian rhythms has been shown in some Dipteran species (Table 1). Moreover, one of four Scandinavian isofemale strains of Drosophila subobscura was arrhythmic, whereas the others had circadian eclosion rhythms. Crosses between the arrhythmic and normal rhythmic strains suggested that arrhythmicity is caused by mutation at one locus on an autosome (Lankinen, 1993a). In the melon fly, Bactrocera cucurbitae, artificial selection for life history traits caused variation in the circadian period of adult locomotor activity. In this species, the circadian period was approximately 22 and $26-31 \mathrm{~h}$ in the lines selected for shorter and longer developmental period, respectively (Shimizu et al., 1997). Similarly, it was approximately 22-25 and 26-28 h in the lines selected for younger and older age at reproduction, respectively (Miyatake, 2002). Crosses between the lines with shorter and longer periods suggested that the circadian rhythm is under 
Table 1 Geographic variation in circadian period $(\tau)$ in Dipterans

\begin{tabular}{|c|c|c|c|}
\hline Species & Trait & Variation in $\tau$ & Reference \\
\hline Drosophila littoralis & Eclosion & $5 \mathrm{~h}$ & Lankinen (1986) \\
\hline Drosophila auraria & Eclosion & $0.5 \mathrm{~h}$ & Pittendrigh and Takamura (1989) \\
\hline Drosophila subobscura & Eclosion & $2.2 \mathrm{~h}$ & Lankinen (1993b) \\
\hline \multirow[t]{3}{*}{ Drosophila ananassae } & Eclosion & $3 \mathrm{~h}$ & Joshi and Gore (1999) \\
\hline & Adult locomotor activity & $3 \mathrm{~h}$ & Joshi (1999) \\
\hline & Oviposition & $5 \mathrm{~h}$ & Khare et al. (2005) \\
\hline Culex pipiens complex & Adult flight or locomotor activity & $3 \mathrm{~h}$ & Shinkawa et al. (1994) \\
\hline
\end{tabular}

Table 2 The rearing history of Callosobruchus chinensis strains used in this study

\begin{tabular}{llcll}
\hline Strain & Locality of population & Collection date & Number of founder adults & Reference \\
\hline jC-S & Kyoto, Kyoto, Japan & 1936 & Unknown & Utida (1941a, b) \\
mC & Morioka, Iwate, Japan & $1960 \mathrm{~s}$ & Unknown & Nakamura (1969) \\
isC & Ishigaki, Okinawa, Japan & 1997 & About 200 & Yanagi and Miyatake (2003) \\
rdaCmrkt & Rajshahi, Bangladesh & 1998 & More than 50 & Harano and Miyatake (2007) \\
yoC02 & Akaiwa, Okayama, Japan & 2002 & 26 & Harano and Miyatake (2005) \\
smC02 & Izumo, Shimane, Japan & 2002 & More than 20 & Harano and Miyatake (2005) \\
kiC07 & Inami, Hyogo, Japan & 2007 & More than 100 & No \\
\hline
\end{tabular}

the control of a major gene on an autosome in $B$. cucurbitae (Shimizu et al., 1997). In the wing-polymorphic cricket, Gryllus firmus, endocrine circadian rhythm differed between the lines selected for long- and shortwinged morphs (Zera and Zhao, 2009).

A number of strains of the adzuki bean beetle, Callosobruchus chinensis (Coleoptera: Bruchidae), have been maintained in isolation from any gene flow in the laboratory (for example, Harano and Miyatake, 2005). In this study, we found marked variation in circadian rhythms of adult locomotor activity between the strains of $C$. chinensis. We then carried out crosses between different strains to analyze the inheritance of circadian rhythm in C. chinensis.

\section{Materials and methods}

\section{Study organisms}

C. chinensis is a broad-based pest of stored legumes, such as the adzuki bean, Vigna angularis and the cowpea, Vigna unguiculata (Kiritani, 1961). Adult females lay eggs on the surface of host beans. Hatched larvae burrow into the bean in which they complete their development, pupation and eclosion. As adults can reproduce without feeding, in the laboratory the adults generally have access to neither food nor water and were simply provided with beans as oviposition substrate.

We used seven strains of $C$. chinensis that originated from beetles collected at different times and sites (Table 2). Before experiments, stock cultures of these strains had been maintained as mass cultures on adzuki beans under a $25^{\circ} \mathrm{C}, 50 \%$ relative humidity and with a photoperiod cycle of 14:10 h light-dark conditions.

\section{Monitoring circadian rhythm}

Beans containing developing larvae were maintained under a $25^{\circ} \mathrm{C}, 60 \%$ relative humidity and photoperiod cycle of 16:8 h light-dark conditions. On emergence, all beetles used were virgin males under 24-h post-emer- gence from bean. The beetles were placed individually in Petri dishes (30 mm diameter, $15 \mathrm{~mm}$ height) under a $25{ }^{\circ} \mathrm{C}, 60 \%$ relative humidity and DD (constant darkness) condition. Their locomotor activities were monitored by interruptions of an infrared beam that vertically crossed the inside of the Petri dish and was connected to a photoelectric switch (E3R-5E4 Omron, Kyoto, Japan). Interruption signals were sent to a computer (BGD246CM1 Epson, Tokyo, Japan) and their numbers were recorded. The free-running period, $\tau$, was calculated from the free-running data of locomotor activities of 10 consecutive days after a beetle had been transferred to the DD condition using a $\chi^{2}$ periodogram (Sokolove and Bushell, 1978). If a beetle died within 10 days, the $\tau$ was calculated from the free-running data of the days before the termination of the locomotor activity record. Beetles that had free-running data of $<5$ days were excluded from all analyses because their data are insufficient to calculate $\tau$. In the $\chi^{2}$ periodogram, the statistic, $Q_{p}$, which depends on the amplitude, was calculated for a given test period in 0.1-h intervals, and the period with highest $Q_{p}$ was considered the free-running period. The individuals for which the highest $Q_{p}$ was below statistical significance level were considered arrhythmic.

\section{Crosses}

We made reciprocal crosses of two combinations of strains. To establish each cross, we placed 5-10 virgin females and 5-10 virgin males in a Petri dish $(90 \mathrm{~mm}$ diameter, $15 \mathrm{~mm}$ height) containing adzuki beans as the oviposition substrate and allowed them to mate and oviposit for 1 day. Cross 1 was between smC02, which had the shortest $\tau$ and was frequently arrhythmic, and jC-S, which had the longest $\tau$, strains. Cross 2 was between yoC02, which had the shortest $\tau$ among the strains that $\tau$ were detected in almost all beetles, and jC-S. In the first $\left(F_{1}\right)$ and second $\left(F_{2}\right)$ generations of the crosses, the circadian rhythm of adult locomotor activity was examined as described above. 
Statistical analysis

To compare the proportion of arrhythmic individuals between strains or between parental strains and $F_{1}$ of reciprocal crosses, we used logistic regression, assessing the significance using a likelihood ratio $\chi^{2}$-test. The significance level for multiple comparisons was corrected by the sequential Bonferroni method (Holm, 1979). To compare $\tau$ between strains or between parental strains and $\mathrm{F}_{1}$ of reciprocal crosses, we used the Tukey's HSD method. To test correlation of $\tau$ calculated from the two measures (see Results), we used Pearson's productmoment correlation. All these statistical analyses were carried out using JMP version 7 (SAS Institute, 2007). According to the joint scaling test (Lynch and Walsh, 1998), the additive and dominance components in the inheritance of $\tau$ were estimated from the results of crosses between strains.

\section{Results}

\section{Comparisons between strains}

Actograms of individuals that had average free-running rhythms in each strain are shown in Figure 1. The proportion of arrhythmic individuals differed significantly between the strains, ranging from 0 to approximately $90 \%$ (Table 3 ). The proportion in each of the three low strains (isC, yoC02 and jC-S), $<5 \%$, was significantly lower than that in each the three high strains ( $\mathrm{smC02,} \mathrm{mC}$ and $\mathrm{rdaCmrkt)}$, and it was also significantly lower in the kiC07 strain than in the smC02 and rdaCmrkt strains. The number of days in which locomotor activities were recorded differed among individuals, ranging from 5 to 10 , as many beetles died before completing 10 days. To eliminate the possibility that differences in the number of days were responsible for differences in the proportion of arrhythmic individuals between strains, we recalculated the free-running rhythm for all beetles using free-running data of simply the first 5 days. The differences in the proportion of arrhythmic individuals between strains remained significant when we did so, with the exception that the $\mathrm{mC}$ strain did not differ from the isC, yoC02 and jC-S strains. The recalculated $\tau$ was significantly correlated with the $\tau$ calculated from all free-running data that was recorded $(r=0.642, P<0.001)$.

The mean $\tau$ varied from approximately 19 to $23 \mathrm{~h}$ between the strains (Table 4). Two strains, $\mathrm{mC}$ and rdaCmrkt, were excluded from the comparison of $\tau$, as for these strains $\tau$ was detected in only a few individuals. There were significant differences in $\tau$ between the strains. The $\tau$ in the shortest strain (smC02) differed significantly from that in each the two long strains (kiC07 and $\mathrm{jC}-\mathrm{S}$ ), and the $\tau$ in the longest strain (jC-S) differed significantly from that in any other strain.

\section{Crosses}

The free-running rhythm patterns in the $F_{1}$ and $F_{2}$ generations were similar in the two crosses (Figures 2 and 3). No $F_{1}$ beetles were arrhythmic in both crosses (Figures $2 b$ and $3 b$ ). In Cross 1 , the $\tau$ of both $F_{1}$ from reciprocal crosses ( $\mathrm{smC} 02$ females $\times \mathrm{jC}-\mathrm{S}$ males: mean \pm s.e. $=21.1 \pm 0.1 \mathrm{~h}$; jC-S females $\times$ smC02 males: mean \pm s.e. $=20.8 \pm 0.2 \mathrm{~h}$ ) differed significantly from each parental strain (see Table 4 ), and the $\tau$ did not differ significantly between $F_{1}$ from reciprocal crosses (Tukey's

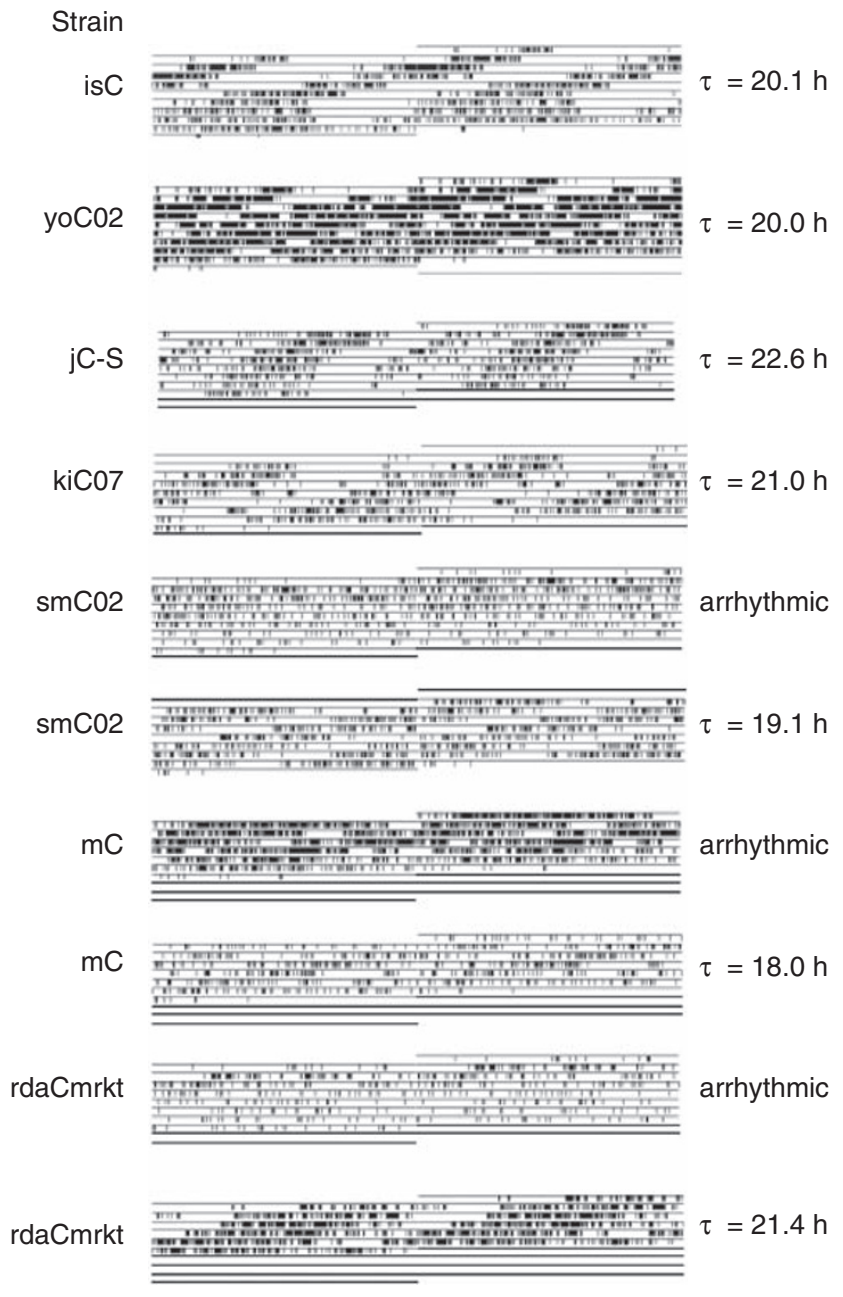

Figure 1 Typical actograms of adult locomotor activity in each strain under the DD condition. They belong to one beetle that showed the average free-running period $(\tau)$ in the upper four strains and two beetles that were arrhythmic and showed average freerunning period in the lower three strains in which most beetles were arrhythmic. Actograms are double-plotted. The free-running rhythms are shown on the right.

Table 3 Proportion of arrhythmic individuals in each strain

\begin{tabular}{lcc}
\hline Strain & $\mathrm{N}$ & Arrhythmic (\%) \\
\hline isC & 19 & $0.0 \mathrm{a}$ \\
yoC02 & 19 & $0.0 \mathrm{a}$ \\
jC-S & 21 & $4.8 \mathrm{a}$ \\
kiC07 & 19 & $15.8 \mathrm{ab}$ \\
smC02 & 21 & $66.7 \mathrm{c}$ \\
mC & 7 & $71.4 \mathrm{bc}$ \\
rdaCmrkt & 9 & $88.9 \mathrm{c}$
\end{tabular}

Different letters indicate significant differences at $P<0.05$ by logistic regression; the significance level for multiple comparisons was corrected by sequential Bonferroni method (Holm, 1979).

HSD method, $P<0.05)$. Also in Cross 2 , the $\tau$ of both $F_{1}$ from reciprocal crosses (yoC02 females $\times$ jC-S males: mean \pm s.e. $=21.3 \pm 0.2 \mathrm{~h}$; jC-S females $\times$ yoC02 males: mean \pm s.e. $=21.3 \pm 0.2 \mathrm{~h}$ ) differed significantly from each parental strain (see Table 4 ), and the $\tau$ did not differ significantly between $\mathrm{F}_{1}$ from reciprocal crosses (Tukey's HSD method, $P<0.05)$. In both crosses, because there 
Table 4 Free-running period $(\tau)$ of each strain

\begin{tabular}{lcc}
\hline Strain & N & $\tau(h)$ \\
\cline { 3 - 3 } & & Mean \pm s.e. \\
\hline isC & 19 & $20.0 \pm 0.2 \mathrm{ab}$ \\
yoC02 & 19 & $20.1 \pm 0.4 \mathrm{ab}$ \\
jC-S & 20 & $22.6 \pm 0.2 \mathrm{c}$ \\
kiC07 & 16 & $21.0 \pm 0.3 \mathrm{~b}$ \\
smC02 & 7 & $18.7 \pm 0.3 \mathrm{a}$ \\
mC & 2 & $18.8 \pm 0.8^{\mathrm{a}}$ \\
rdaCmrkt & 1 & $21.4^{\mathrm{a}}$ \\
\hline
\end{tabular}

Different letters indicate significant differences at $P<0.05$ by the Tukey's HSD method.

${ }^{a}$ These two strains were excluded from comparison because of being of extremely small sample size.
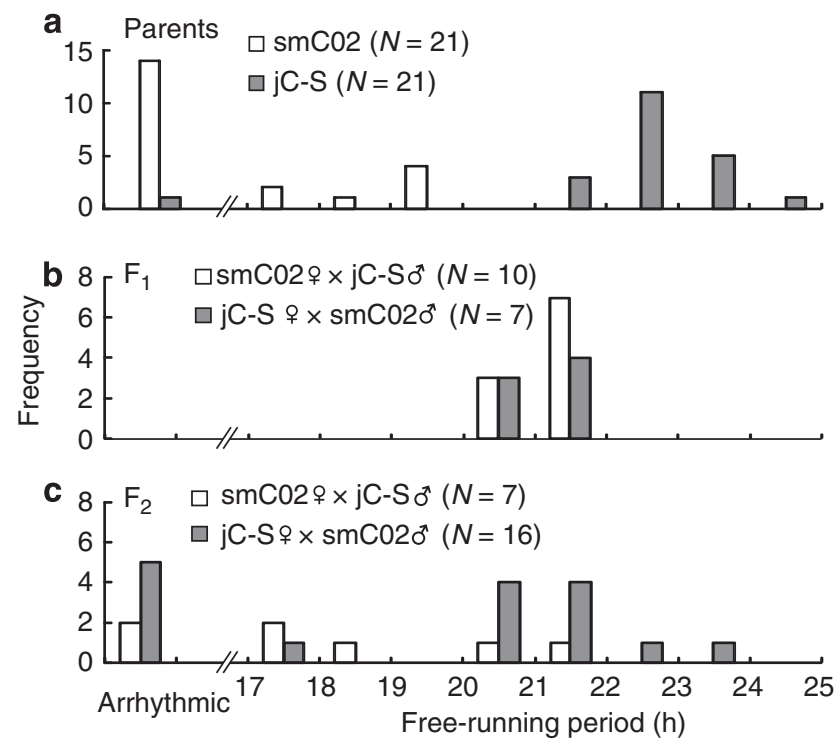

Figure 2 Frequency distributions of free-running rhythms in the parental strains (a) and their $\mathrm{F}_{1}(\mathbf{b})$ and $\mathrm{F}_{2}(\mathbf{c})$ in Cross 1: the smC02 and jC-S strains.

were no differences in $\tau$ between $F_{1}$ from reciprocal crosses, we pooled the data from reciprocal crosses in the following analyses. The $\tau$ of all $F_{1}$ individuals fell between the means of the parental strains in Cross 1 ( $\tau=18.7$ and 22.6 h; Figure $2 b)$ and Cross $2(\tau=20.1$ and $22.6 \mathrm{~h}$; Figure $3 \mathrm{~b}$ ). In contrast, in $\mathrm{F}_{2}$ from Cross $1,30.4 \%$ of beetles were arrhythmic, some of the remaining beetles had a $\tau$ similar to the shorter parental strain, and others had $\tau$ similar to the longer parental strain (Figure $2 \mathrm{c}$ ). In Cross 2, almost all $(95.2 \%) \quad F_{2}$ beetles had statistically significant periodicity, some of them had a $\tau$ similar to the shorter parental strain, and others had a $\tau$ similar to the longer parental strain (Figure 3c). Thus, the segregation of free-running rhythms appeared in the $\mathrm{F}_{2}$ generation of both crosses. These results are consistent with the expectation of a major gene with additive effects and no dominance in the inheritance of $\tau$.

The circadian period was not detected in most individuals of one parental strain (smC02; Figure 2a) or in many $\mathrm{F}_{2}$ individuals (Figure $2 \mathrm{c}$ ) in Cross 1 , whereas it was detected in almost all individuals in Cross 2 (Figure 3). In the joint scaling test, we could not collectively deal with the length of circadian period

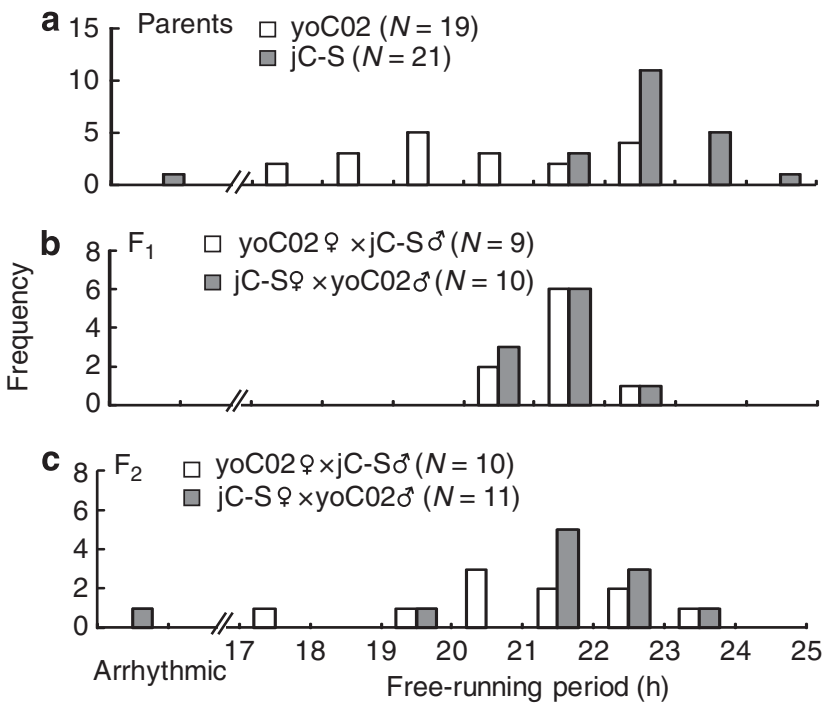

Figure 3 Frequency distributions of free-running rhythms in the parental strains (a) and their $F_{1}$ (b) and $F_{2}$ (c) in Cross 2: the yoC02 and jC-S strains.

and the incidence of circadian rhythm, which are quantitative and qualitative data, respectively. Thus, we subjected only the results of Cross 2 to the joint scaling test, with the exclusion of few arrhythmic individuals, and estimated the parameters of mean $\left(\mu_{0}\right)$, and composite additive $\left(\alpha^{c}\right)$ and dominance $\left(\delta^{c}\right)$ effects (Lynch and Walsh, 1998) in the inheritance of $\tau$. We first assumed the additive model. This model yielded estimates \pm s.e. of $\hat{\mu}_{0}=21.29 \pm 0.10$ and $\hat{\alpha}^{c}=-1.29 \pm 0.17$, and did not significantly differ from the data $\left(\chi^{2}=0.079\right.$, $P=0.961)$, indicating that additive effects adequately explain the inheritance of $\tau$. To confirm the absence of a dominance effect, we evaluated the additive-dominance model. This model yielded the estimates \pm s.e. of $\hat{\mu}_{0}=21.30 \pm 0.11, \hat{\alpha}^{c}=-1.25 \pm 0.20$ and $\hat{\delta}^{c}=-0.03 \pm 0.12$. The $\hat{\mu}_{0}$ and $\hat{\alpha}^{\mathrm{c}}$ were extremely similar to those obtained from the additive model, and the inclusion of dominance in the model did not significantly improve the fit for the data $\left(\chi^{2}=0.0007, P=0.9997\right)$, indicating no dominance in the inheritance of $\tau$.

\section{Discussion}

We found significant differences between strains of C. chinensis in the tendency to express circadian rhythm (Table 3) and the length of the circadian period (Table 4) of adult locomotor activity. This indicates genetic variation in attributes or features of circadian rhythms. The $\tau$ of most $C$. chinensis strains (Table 4) were not long enough to be in the $22-28 \mathrm{~h}$ range that covers the $\tau$ of activity rhythms in many animal species (Lohmann, 1967; Aschoff, 1981; Saunders, 2002). The shortest $\tau$ among the $C$. chinensis strains (Table 4) matched the per $(\tau=19 \mathrm{~h}$ ) of $D$. melanogaster (Konopka and Benzer, 1971) and the strain with shortest circadian period $(\tau=18.8 \mathrm{~h})$ of 27 strains of Drosophila littoralis (Lankinen, 1986).

Major genes altering circadian rhythm have been found primarily within the Diptera. Key examples of such include Drosophila (Konopka and Benzer, 1971; Lankinen, 1993a) and the melon fly, B. cucurbitae (Shimizu et al., 1997). Our study indicated that major 
genes dramatically alter the circadian rhythm in C. chinensis, belonging to a taxon other than Diptera. The absence of differences in circadian rhythm between the $F_{1}$ males from reciprocal crosses (Figures $2 b$ and $3 b$ ) indicates that the major gene altering circadian rhythm locates on an autosome in $C$. chinensis as sex chromosomes of this species are male heterogametic XY (Kondo et al., 2002). This is similar to the major genes controlling the circadian rhythm in D. subobscura (Lankinen, 1993a) and B. cucurbitae (Shimizu et al., 1997), but differs from the location of the per locus in D. melanogaster (Konopka and Benzer, 1971).

In D. melanogaster, heterozygote tests suggested dominance relations between the normal period, long period $\left(p e r^{\mathrm{L}}\right)$ and short period $\left(p e r^{\mathrm{S}}\right)$ alleles at the per locus (Konopka and Benzer, 1971). In the test, the normal allele was observed as dominant to the per $^{\mathrm{L}}$ but only partially dominant to the $p e r^{\mathrm{s}}$, and the $p e r^{\mathrm{L}}$ and $p e r^{\mathrm{s}}$ alleles were revealed to have incomplete dominance. Crosses between lines suggested that a shorter circadian period is partially dominant to a longer one in B. cucurbitae (Shimizu et al., 1997). In contrast to these species, observations and analyses of the $F_{1}$ and $F_{2}$ from crosses between different strains (Figures 2 and 3 ) revealed that the major gene responsible for variation in the period length of circadian rhythm has additive effects and no dominance in $C$. chinensis. At the per locus in D. melanogaster, the normal allele, per $^{\mathrm{L}}$ and per $^{\mathrm{S}}$ are dominant to arrhythmic $\left(\right.$ per $\left.^{\circ}\right)$. In D. subobscura, normal circadian eclosion rhythm is dominant to arrhythmicity (Lankinen, 1993a). In C. chinensis, arrhythmic beetles were not found in the $F_{1}$ from the cross between the smC02 strain in which most beetles were arrhythmic and the jC-S strain in which very few beetles were arrhythmic (Figure 2). This indicates that the expression of the circadian rhythm is dominant to arrhythmicity. However, no strains of $C$. chinensis were absolutely arrhythmic (Table 3). In this study, it should be noted that whether an individual is arrhythmic was determined by one criterion. The dominance of the expression of circadian rhythm may be attributable to the criterion. According to a $\chi^{2}$ periodogram (Sokolove and Bushell, 1978), individuals were classified as arrhythmic if the statistic that depends on the amplitude of rhythmicity was below significance level. In the strains that had many arrhythmic individuals, the amplitude of rhythmicity was generally small even in individuals that were not classified as arrhythmic, because there was no discontinuity in the distribution of amplitude of rhythmicity. The parental strain in which most beetles were arrhythmic (smC02) in the cross showed an extremely short circadian period (Figure 2a). Thus, smaller amplitude may be associated with shorter circadian period in C. chinensis. In this case, the tendency to be arrhythmic should be controlled by genes underlying the length of circadian period, which have additive effects and no dominance.

The genetic variation found in circadian rhythms between the strains of $C$. chinensis could be due to random factors and/or some selection. Random factors include a founder effect and genetic drift. In the first case, the circadian rhythm of each strain may depend on the major gene carried by founder beetles. In the second, a certain major gene controlling circadian rhythm might be fixed in each strain through genetic drift in the wild or during successive rearing in the laboratory.
The strains used for this study differed in their geographical origins (Table 2), thus they should have been subjected to different selective pressures. They also differed in their rearing periods in the laboratory (Table 2), during which the insects would be subjected to some selection (Miyatake, 1998). Thus, selection history differed between the strains. Selection for circadian rhythm may result in adaptive divergence in this trait between the strains. Owing to their ubiquity, circadian rhythms are believed to evolve through natural selection (Sharma, 2003), although only a few studies have shown a selective advantage attached to circadian rhythm. In Arabidopsis, plants that had a circadian period closer to the period of external light-dark cycle grew faster and survived better (Dodd et al., 2005). Strains of cyanobacteria that had a circadian period more similar to the period of the external light-dark cycle achieved a higher growth rate when competing with other strains (Ouyang et al., 1998).

Selection acting on traits genetically correlated with circadian rhythm, instead of acting on the circadian rhythm itself, may cause genetic divergence in circadian rhythm between the strains of $C$. chinensis. The genes controlling circadian rhythm affect other traits such as male courtship song (Kyriacou and Hall, 1980), development time (Kyriacou et al., 1990; Miwa and Yajima, 1995; Wong et al., 1995), and adult longevity (Lakowski and Hekimi, 1996; Hurd and Ralph, 1998; Klarsfeld and Rouyer, 1998). Selection for such traits should produce a correlated response in circadian rhythm. In B. cucurbitae, artificial selection for development time generated variations in the period of circadian rhythm of approximately $8 \mathrm{~h}$ (Shimizu et al., 1997) and similar selection for age at reproduction produced variations of $6 \mathrm{~h}$ (Miyatake, 2002) as correlated responses. Further study is required to test genetic correlations between circadian rhythm and other traits in C. chinensis.

\section{Conflict of interest}

The authors declare no conflict of interest.

\section{Acknowledgements}

We thank Yukihiko Toquenaga (Tsukuba University, Ibaraki, Japan), Masakazu Shimada (University of Tokyo, Tokyo, Japan), Natsuko Kondo (National Institute for Environmental Studies, Ibaraki, Japan), Katsuyuki Kohno (National Institute of Vegetable and Tea Science, Mie, Japan), Yutaka Narai (Shimane Agricultural Experiment Station, Shimane, Japan) and Yukari Matsubara (Hyogo Prefecture Nishiwaki Agricultural Improve Propagate Center, Hyogo, Japan) for providing the insect cultures of the bean beetles. We also thank Kozue Hatashi (Okayama University, Okayama, Japan) for technical advice, Chris Wood (GCOE, Kyushu University, Fukuoka, Japan) for checking through the paper, Akira Matsumoto (Juntendo University, Chiba, Japan) for useful suggestions and anonymous referees for valuable comments. This study was partly supported by Grant-in-Aid for JSPS Fellows (203976) to TH and Grant-in-Aid for Scientific Research (KAKENHI 19370011) to TM, both from the Japan Society for the Promotion of Science. 


\section{References}

Aschoff J (1981). Handbook of Behavioral Neurobiology, Vol.4 Biological Rhythms. Plenum Press: New York.

Bruce VG (1972). Mutants of the biological clock in Chiamydomonas reinhardi. Genetics 70: 537-548.

Dodd AN, Salathia N, Hall A, Kévei E, Tóth R, Nagy F et al. (2005). Plant circadian clocks increase photosynthesis, growth, survival, and competitive advantage. Science 309: 630-633.

Edmunds LN (1988). Cellular and Molecular Bases of Biological Clocks: Models and Mechanisms for Circadian Timekeeping. Springer-Verlag: New York.

Feldman JF, Hoyle MN (1973). Isolation of circadian clock mutants of Neurospora crassa. Genetics 75: 605-613.

Hall JC (2003). Genetics and molecular biology of rhythms in Drosophila and other insects. Adv Genet 48: 1-280.

Harano T, Miyatake T (2005). Heritable variation in polyandry in Callosobruchus chinensis. Anim Behav 70: 299-304.

Harano T, Miyatake T (2007). Interpopulation variation in female remating is attributable to female and male effects in Callosobruchus chinensis. J Ethol 25: 49-55.

Holm S (1979). A simple sequentially rejective multiple test procedure. Scand J Stat 6: 65-70.

Hurd MW, Ralph MR (1998). The significance of circadian organization for longevity in the Golden hamsters. J Biol Rhythm 13: 430-436.

Joshi DS (1999). Latitudinal variation in locomotor activity rhythm in adult Drosophila ananassae. Can J Zool 77: 1-6.

Joshi DS, Gore AP (1999). Latitudinal variation in eclosion rhythm among strains of Drosophila ananassae. Indian J Exp Biol 37: 718-724.

Khare PV, Satralkar MK, Vanlalnghaka C, Keny V, Lkastur MS, Shivagaje AJ et al. (2005). Altitudinal variation in the circadian rhythm of oviposition in Drosophila ananassae. Chronobiol Int 22: 45-57.

Kiritani K (1961). The origin of household pests, and the process of adaptation to indoor conditions. Seitai Kontyu 9: 22-40 (in Japanese).

Klarsfeld A, Rouyer F (1998). Effects of circadian mutations and LD periodicity on the life span of Drosophila melanogaster. I Biol Rhythm 13: 471-478.

Kondo N, Nikoh N, Ijichi N, Shimada M, Fukatsu T (2002). Genome fragment of Wolbachia endosymbiont transferred to X chromosome of host insect. Proc Natl Acad Sci USA 99: 14280-14285.

Kondo T, Tsinoremas NF, Golden SS, Johnson CH, Kutsuna S, Ishiura M (1994). Circadian clock mutants of cyanobacteria. Science 266: 1233-1236.

Konopka R, Benzer S (1971). Clock mutants of Drosophila melanogaster. Proc Natl Acad Sci USA 68: 2112-2116.

Kyriacou CP, Hall JC (1980). Circadian rhythm mutations in Drosophila affect short-term fluctuations in the male's courtship song. Proc Natl Acad Sci USA 77: 6929-6933.

Kyriacou CP, Oldroyd M, Wood J, Sharp M, Hill M (1990). Clock mutations alter developmental timing in Drosophila. Heredity 64: 395-401.

Lakowski B, Hekimi S (1996). Determination of life-span in Caenorhabditis elegans by four clock genes. Science 272: 1010-1013.

Lankinen P (1986). Geographical variation in circadian eclosion rhythm and photoperiodic adult diapause in Drosophila littoralis. J Comp Physiol A 159: 123-142.

Lankinen P (1993a). Characterization of linne, a new autosomal eclosion rhythm mutant in Drosophila subobscura. Behav Genet 23: 359-367.

Lankinen P (1993b). North-south differences in circadian eclosion rhythm in European populations of Drosophila subobscura. Heredity 71: 210-218.

Lohmann M (1967). Ranges of circadian period length. Cell Mol Life Sci 23: 788-790.

Lynch M, Walsh B (1998). Genetics and Analysis of Quantitative Traits. Sinauer Associates: Sunderland.
Matsumoto A, Ohta Y, Itoh TQ, Sanada-Morimura S, Matsuyama T, Fuchikawa T et al. (2008). Period gene of Bactrocera cucurbitae (Diptera: Tephritidae) among strains with different mating times and sterile insect technique. Ann Entomol Soc Am 101: 1121-1130.

Millar AJ, Carré IA, Strayer CA, Chua NH, Kay SA (1995). Circadian clock mutants in Arabidopsis identified by luciferase imaging. Science 267: 1161-1163.

Miwa I, Yajima H (1995). Correlation of the period length of circadian rhythms with the length of immaturity in Paramecium bursaria. Zool Sci 12: 53-59.

Miyatake T (1998). Genetic changes of life history and behavioral traits during mass-rearing in the melon fly, Bactrocera cucurbitae (Diptera: Tephritidae). Res Popul Ecol 41: 269-273.

Miyatake T (2002). Circadian rhythm and time of mating in Bactrocera cucurbitae (Diptera: Tephritidae) selected for age at reproduction. Heredity 88: 302-306.

Nakamura H (1969). Geographic variation of the ecological characters in Callosobruchus chinensis L. Jpn J Ecol 19: 127-131 (in Japanese with English summary).

Ouyang Y, Andersson CR, Kondo T, Golden SS, Johnson CH (1998). Resonating circadian clocks enhance fitness in cyanobacteria. Proc Natl Acad Sci USA 95: 8660-8664.

Panda S, Hogenesch JB, Kay SA (2002). Circadian rhythms from flies to human. Nature 417: 329-335.

Pittendrigh CS, Takamura T (1989). Latitudinal clines in the properties of a circadian pacemaker. J Biol Rhythm 4: 217-235.

Ralph MR, Menaker M (1988). A mutation of the circadian system in golden hamsters. Science 241: 1225-1227.

Reppert SM, Tsai T, Roca AL, Sauman I (1994). Cloning of a structural and functional homolog of the circadian clock gene period from the giant silkmoth Antheraea pernyi. Neuron 13 1167-1176.

SAS Institute (2007). JMP7. SAS Institute: Cary.

Saunders DS (2002). Insect Clocks. Elsevier Science: Amsterdam.

Sharma VK (2003). Adaptive significance of circadian clocks. Chronobiol Int 20: 901-919.

Shimizu T, Miyatake T, Watari Y, Arai T (1997). A gene pleiotropically controlling developmental and circadian periods in the melon fly, Bactrocera cucurbitae (Diptera: Tephritidae). Heredity 70: 600-605.

Shinkawa Y, Takeda S, Tomioka K, Matsumoto A, Oda T, Chiba Y (1994). Variability in circadian activity patterns within the Culex pipiens complex (Diptera: Culicidae). I Med Entomol 31: 49-56.

Sokolove PG, Bushell WN (1978). The chi square periodogram: its utility for analysis of circadian rhythms. J Theor Biol 72: 131-160.

Tei H, Okamura H, Shigeyoshi Y, Fukuhara C, Ozawa R, Hirose $M$ et al. (1997). Circadian oscillation of a mammalian homologue of the Drosophila period gene. Nature 389: 512-516.

Utida S (1941a). Studies on experimental population of the azuki bean weevil Callosobruchus chinensis (L.). I. The effect of population density on the progeny population. Mem Coll Agric Kyoto Imperial Univ 48: 1-31.

Utida S (1941b). Studies on experimental population of the azuki bean weevil Callosobruchus chinensis (L.). IV. Analysis of density effect with respect to fecundity and fertility of eggs. Mem Coll Agric Kyoto Imperial Univ 51: 1-26.

Vitaterna MH, King DP, Chang AM, Kornhauser JM, Lowrey PL, McDonald JD et al. (1994). Mutagenesis and mapping of a mouse gene, clock, essential for circadian behavior. Science 264: 719-725.

Wong A, Boutis P, Hekimi S (1995). Mutations in the $c l k-1$ gene of Caenorhabditis elegans affect developmental and behavioural timing. Genetics 139: 1247-1259.

Yanagi S, Miyatake T (2003). Costs of mating and egg production in female Callosobruchus chinensis. J Insect Physiol 49: 823-827.

Zera AJ, Zhao Z (2009). Morph-associated JH titer diel rhythm in Gryllus firmus: experimental verification of its circadian basis and cycle characterization in artificially selected lines raised in the field. J Insect Physiol 55: 450-458. 